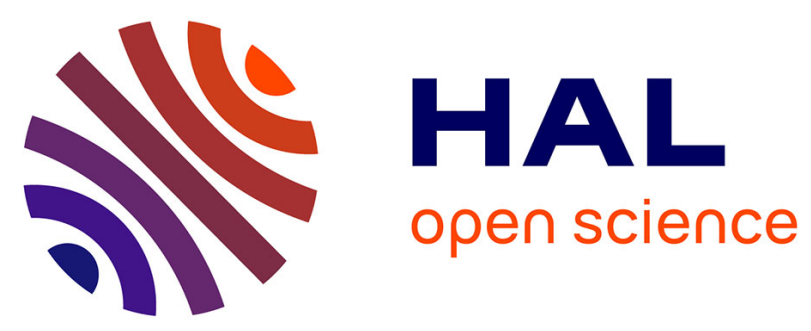

\title{
Detection of Intrinsic Optical Signals in the Somatosensory Cortex of Neonatal Rats by Principal Components Analysis
}

\author{
M. Yu. Sintsov, D. S Suchkov, M. G. Minlebaev
}

\section{To cite this version:}

M. Yu. Sintsov, D. S Suchkov, M. G. Minlebaev. Detection of Intrinsic Optical Signals in the Somatosensory Cortex of Neonatal Rats by Principal Components Analysis. Neuroscience and Behavioral Physiology, 2018, 48 (5), pp.551-556. 10.1007/s11055-018-0598-0 . hal-01963815

\section{HAL Id: hal-01963815 \\ https://hal-amu.archives-ouvertes.fr/hal-01963815}

Submitted on 21 Dec 2018

HAL is a multi-disciplinary open access archive for the deposit and dissemination of scientific research documents, whether they are published or not. The documents may come from teaching and research institutions in France or abroad, or from public or private research centers.
L'archive ouverte pluridisciplinaire HAL, est destinée au dépôt et à la diffusion de documents scientifiques de niveau recherche, publiés ou non, émanant des établissements d'enseignement et de recherche français ou étrangers, des laboratoires publics ou privés.

\section{(c)(1)}

Distributed under a Creative Commons Attribution| 4.0 International License 


\title{
Detection of Intrinsic Optical Signals in the Somatosensory Cortex of Neonatal Rats by Principal Components Analysis
}

\author{
M. Yu. Sintsov, ${ }^{1}$ D. S. Suchkov, ${ }^{1}$ and M. G. Minlebaev ${ }^{1-3}$
}

Translated from Rossiiskii Fiziologicheskii Zhurnal imeni I. M. Sechenova, Vol. 103, No. 2, pp. 152-160, February, 2017. Original article submitted November 25, 2016.

Recording of the intrinsic optical signal (IOS) is widely used or functional studies of the cerebral cortex in vivo. Despite the fact that IOS provides for detection of active areas, regardless of the age of the object, it is widely used in studies of the developing brain. However, in immature brains IOS has low amplitude, which hinders its use and requires other recording and analysis methods. We report here our assessment of the use of the principal components analysis (PCA) method for the automatic detection of IOS at the early stages of development of the rat brain. Recording of IOS in infrared light and use of PCA was found to provide reliable detection of IOS in rats in the first three weeks after birth. Addition of artificial noise to IOS showed that detection using PCA was effective in half of cases despite increases in the noise level to four times baseline. These results provide evidence that the PCA method has potential to be used for detecting IOS at the early stages of development and that the PCA method is very robust for detection of IOS.

Keywords: cortex, intrinsic optical signal, principal components analysis.

Neuroimaging provides a potential direction in neurobiology providing for functional studies in the central nervous system. Among the multitude of different neuroimaging methods, recording of the intrinsic optical signal (IOS) has a number of advantages. In contrast to other methods, IOS has low invasivity and is economical, though it is highly accurate, which makes it the method of choice for functional mapping in the somatosensory, auditory, and visual areas of the cortex. A limitation of the IOS recording method is its relatively low signal:noise ratio $[1,2]$, which hinders automatic detection of IOS and its analysis. We have previously demonstrated positive results using temporal and spatial filters to increase the signal:noise ratio [3], though this did not solve the problem of subjectivity in detection of IOS by the operator. Current analytical algorithms for automatic detection include the use of indicator functions, truncated differences, and independent components analysis

\footnotetext{
${ }^{1}$ Developmental Neurobiology Laboratory, Kazan Federal University, Kazan, Russia.

${ }^{2}$ Institut de Neurobiologie de la Méditerranée, INMED-INSERM U901, Marseille, France.; e-mail: marat.minlebaev@inserm.fr

${ }^{3}$ Aix-Marseille University, Marseille, France.
}

[4-6]. In addition, these methods are energy-intensive and use extensive computation resources; they were developed for detecting IOS in mature neural tissue. As compared with the adult brain, functional mapping of the brain at its early developmental stages involves a significantly smaller set of techniques for detection of active areas in cortical tissue. Thus, the questions of the use of IOS and means for its detection in the developing brain are currently very relevant. The studies reported here demonstrate that use of principal components analysis (PCA) allows automatic detection of IOS at the level of cortical columns in the barrel cortex of neonatal rats in response to stimulation of the corresponding sensory input. PCA has been shown to be effective despite addition of noise to the initial data making IOS visually undetectable.

The aim of the present work was to assess the potential of using the principal components analysis method for automatic detection of active cortical columns at the early stages of development of the brain in rat pups.

Methods. Surgery. All protocols using animal models took account of the methodological recommendations of the French National Institute for Medicine and Health Research (INSERM) (N007.08.01) and the regulations for the use of 

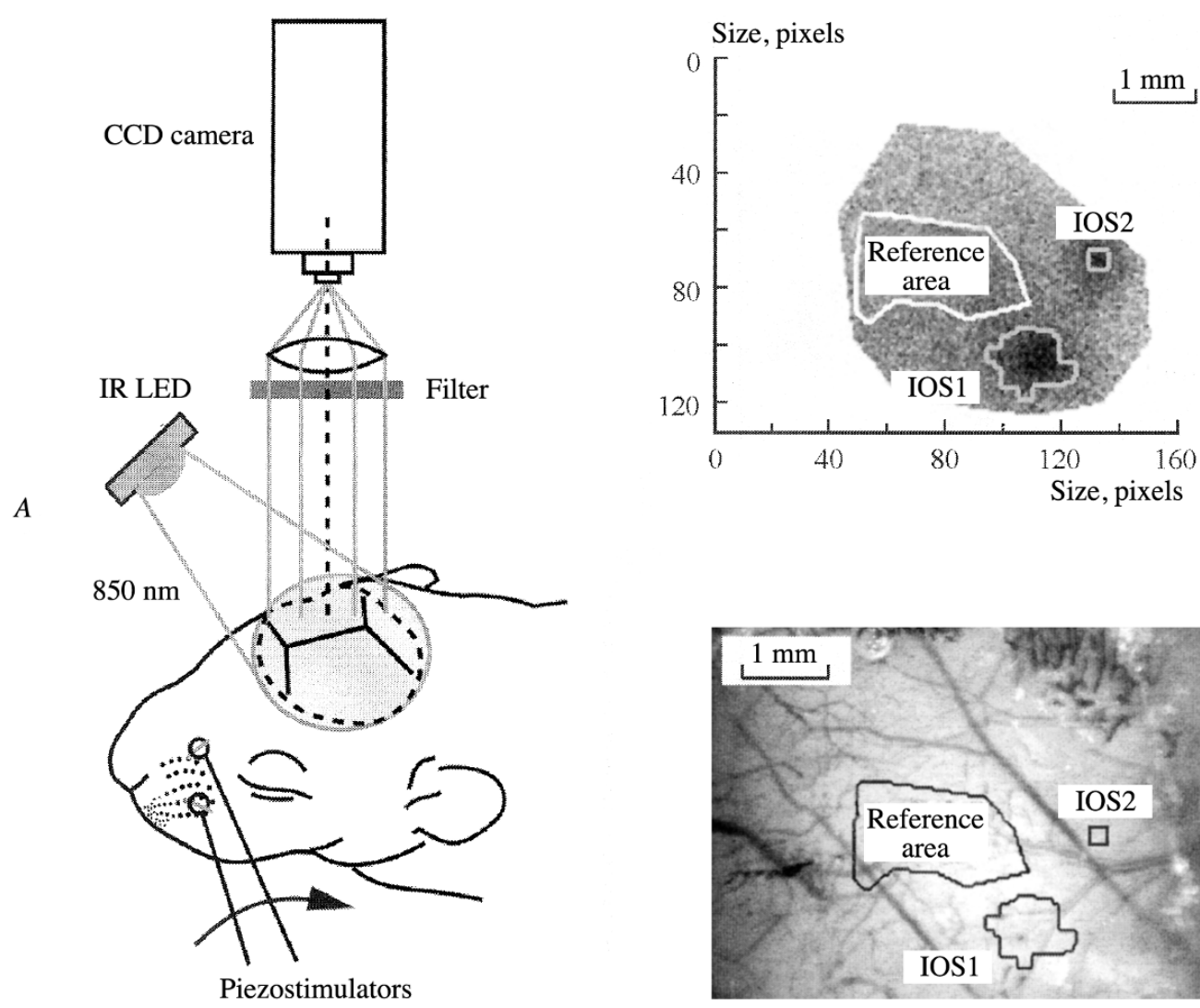

\section{$B$}

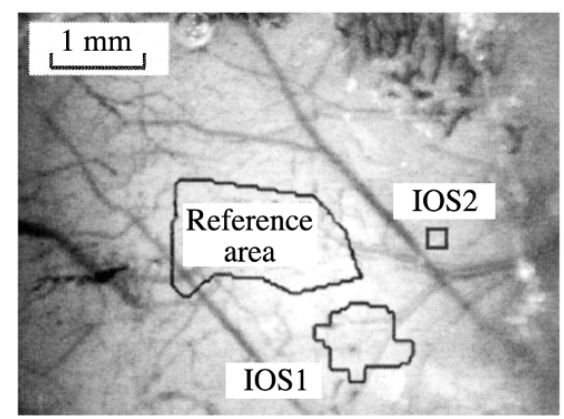

$C$

Fig. 1. Recording of sensory-evoked intrinsic optical signal (IOS) in the somatosensory cortex of a neonatal rat pup. A) Diagram of experimental apparatus; $B$ ) example of automatic detection of intrinsic optical signals in the primary somatosensory cortex (IOS1 and IOS2) evoked by stimulation of two whiskers (A1 and E1) on the rat pup's snout. Left - outlines of reference area used for comparison of IOS parameters; $C$ ) spatial position of IOS and reference area in relation to the network of superficial blood vessels.

laboratory animals of Kazan Federal University (ethical approval, Institute for the Care of Animals, and the Committee Monitoring Animal Use, Kazan State Medical University, N9-2013). Wistar rats both genders of postnatal age P3-P24, where P0 was the day of birth, were used. Surgery was performed under isoflurane anesthesia. The skull was cleaned of skin and periosteum, after which it was covered with dental cement except for an area of $4 \times 9 \mathrm{~mm}$ above the barrel cortex. The animal's head was fixed and immobilized by attaching a metal holder to the cement, the other end of which was mounted in a stereotaxic apparatus. Anesthesia was performed for animals of all ages. During experiments, the animal was warmed to a physiological temperature of $35-37^{\circ} \mathrm{C}$ with a warming blanket and by wrapping in cotton wool.

Stimulation. Before experiments, all sensory whiskers on the animal's snout were trimmed to a length of $\sim 3 \mathrm{~mm}$. A fine wire ring $(\sim 100 \mu \mathrm{m}$ in diameter $)$ was glued to the end of a piezodeflector (Noliak, USA); the end of the whisker was inserted into the ring to a depth of $\sim 2 \mathrm{~mm}$ such the that the whisker was freely located within the ring. The piezodeflector was activated using square-wave electric impulses of duration $10 \mathrm{msec}$ and amplitude 80-90 V. The standard stimulation protocol consisted of $5 \mathrm{sec}$ of reference signal, $10 \mathrm{sec}$ of periodic stimulation, and $55 \mathrm{sec}$ of recovery, totaling $70 \mathrm{sec}$ between repeat stimuli to reduce depression of the evoked response. Cortical responses from 12-20 series of stimulation trials were recorded for each whisker, which was followed by averaging to increase the signal:noise ratio $(\mathrm{S}: \mathrm{N})$. Different stimulation frequencies were used for each age group to elicit maximum-amplitude IOS (the overall stimulation frequency range was $0.1-10 \mathrm{~Hz}$ ).

The intrinsic optical signal. IOS was recorded (Fig. 1) using a video camera positioned perpendicular to the bare skull surface above the presumptive position of the barrel cortex [7]. High-contrast images of the surface network of blood vessels were obtained with illumination with green light (528 nm), using a light-emitting diode (Arlight, China). In vivo IOS recordings were made using light in the near infrared $(850 \mathrm{~nm})$ and the focus of the optical system of the video camera was established at a depth corresponding to the granular layer of the barrel cortex [8]. Reflected light from the illuminated surface of the skull, passing through the 


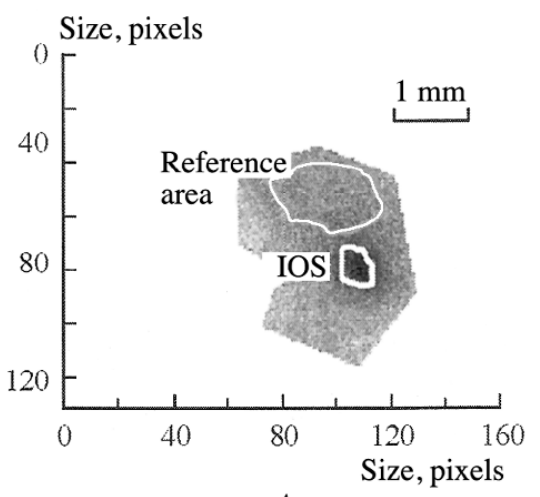

$A$

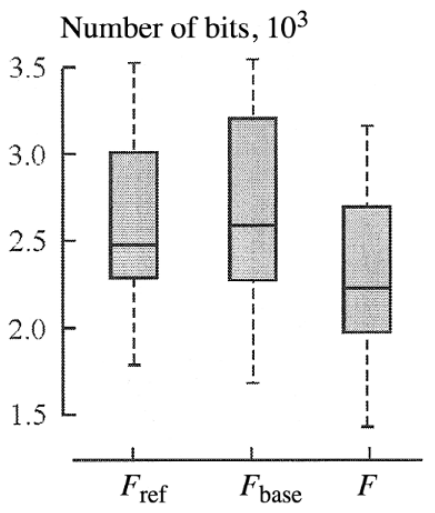

C

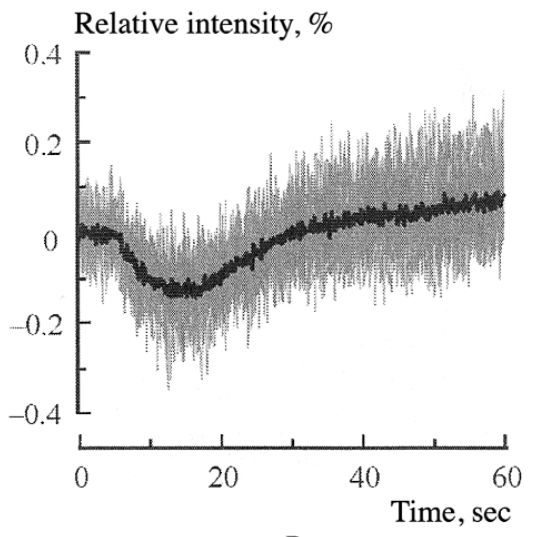

$B$
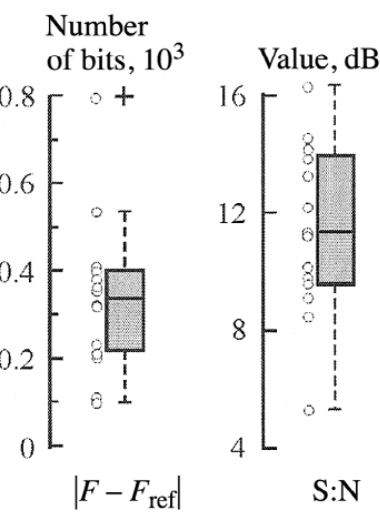

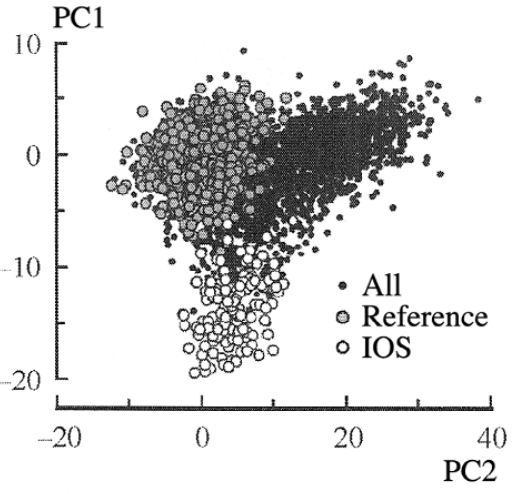

$E$

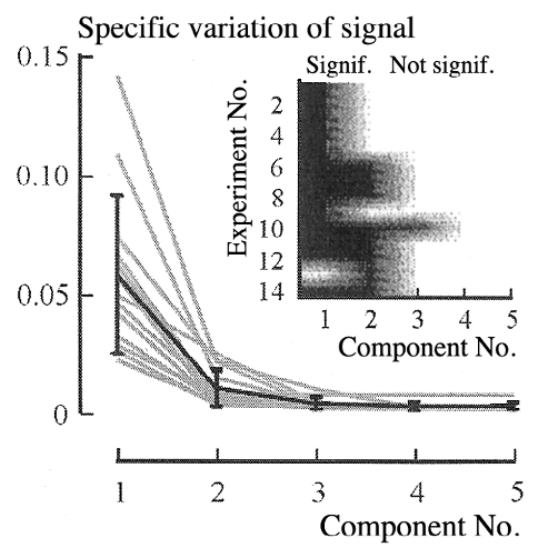

$F$

Fig. 2. Principles of detection of IOS using the principal components analysis method. A) IOS detected using the PCA method (white outline) - the reference area is marked in gray; the age of the animal was P6; $B$ ) time course of IOS expressed as changes in the intensity of pixels in the IOS positive area shown in $A$; stimulation of a whisker was produced using a volley of mechanical stimuli with a frequency of $5 \mathrm{~Hz}$ in the interval from 5 to $15 \mathrm{sec}$; $C$ ) absolute intensity value for the reference signal $\left(F_{\text {ref }}\right)$, the baseline signal $\left(F_{\text {base }}\right)$, and IOS $\left.(F) ; D\right)$ distribution and group statistics of the difference between the IOS and reference areas, signal:noise ratio for IOS and its relative intensity (RI) $(n=14) ; E)$ distribution of the values of the first two components of pixels included in the analysis (principal components PC1 and PC2) (black circles); the gray area shows pixels in the reference area and the white area shows IOS pixels; $F$ ) averaged relationship between specific variation of the signal and component No. (black line), by default showing only the first three components for detection; individual experiments are shown in gray; the subplot shows the qualitative relationship between components assessed using the broken stick model.

optical focusing system and a set of optical filters (Rosco, China), was detected using a charge-coupled device (resolution $130 \times 174$ points, where 1 point $\sim 35 \mu \mathrm{m}$. Video images of the illuminated cortex were recorded at frequency of 20-50 fps with a QICAM Fast 1394 video camera. For subsequent analysis the frame rate was decreased to $10 \mathrm{~Hz}$.

Artifacts associated with the light source and micromovements of the animal's brain were removed using spatial and temporal filtration as described in our previous report [3]. IOS was detected by comparing averaged video images with before stimulation (F0) with averaged video images during stimulation (F1) $[9,10]$. IOS amplitude was calculated as $\mathrm{A}=(\mathrm{F} 1-\mathrm{F} 0) / \mathrm{F} 0$. Areas of the cerebral cortex showing significant differences in reflected light (more than three standard deviations) were defined as areas of IOS.
Use of PCA to detect IOS. IOS was detected automatically using threshold signal clipping and reconstruction using the PCA method. The signal from the camera was transformed into a matrix in which rows showed values for light intensity in each pixel and columns showed time counts. The matrix was then analyzed by PCA and was sorted in terms of intrinsic number values. The number of components needed to explain the initial variation of the signal was assessed using the broken stick model, which led to selection of three main components explaining $10 \%$ of the initial signal. The amplitude defined as the mean intensity during stimulation was calculated in the reconstructed signal. Significant IOS was identified using a threshold of $2.5 \%$, which corresponds to a significance level of $\alpha=0.05$. In addition, the spatial positions of pixels where the ampli- 


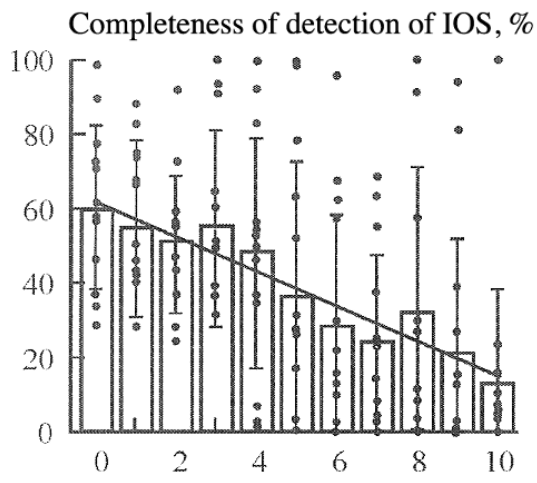

A

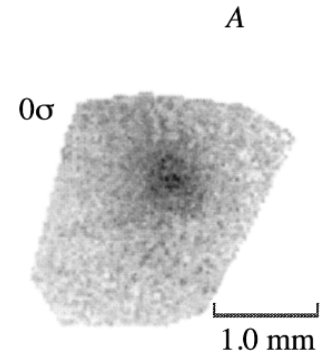

Accuracy of detection, IOS, $\%$

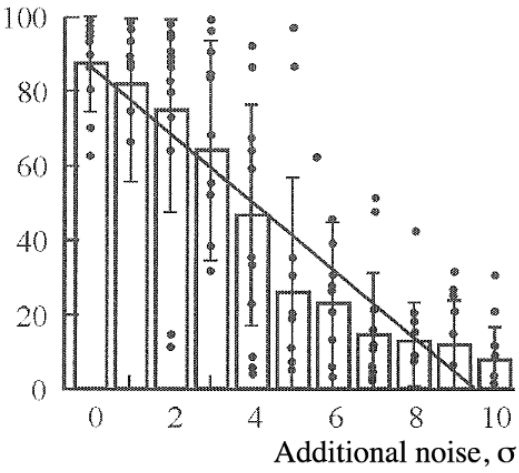

$B$
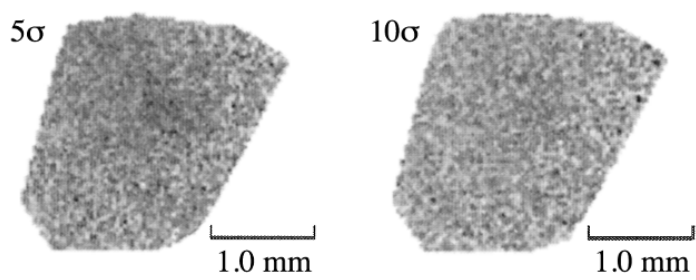

C

Fig. 3. Stability of PCA detection of IOS on artificial addition of noise to image. A) Completeness of detection, reflecting coincidence of the sizes of areas of IOS detected by the observer in control conditions and using PCA at different levels of added noise; $B$ ) accuracy of detection of IOS, characterizing the spatial accuracy of IOS detected using PCA into the IOS area noted by the operator in control conditions. Vertical lines show standard deviations $(n=14)$. The sloped lines show the linear regression describing the mean value of the characteristic. The linear regression intersects the null point at $13 \sigma$ when IOS detection was accurate. $0 \sigma$ corresponds to real data not subjected to additional synthetic increases in noise; $C$ ) examples of synthetic addition of noise the $0 \sigma, 5 \sigma$, and $10 \sigma$ superimposed on the experimental results.

tude of the reconstructed signal exceeded the selected threshold were determined (Fig. 2). The resulting pixel indexes formed a mask for the area of IOS in which the actual amplitudes before and after stimulation were calculated (Fig. 2). Additional verification of the applicability of the method of detecting IOS using PCA was obtained by an operator verifying the results by manual preparation of the mask for the area of IOS. The results of manual determination were then used to assess the effectiveness of automatic detection of IOS.

Results. Recording of IOS using infrared illumination. A series of experiments was performed on 16 animals aged P3-24, in 15 of which IOS was detected using near infrared light. In parallel with analysis of the mean IOS amplitude, which was $2237 \pm 128$ bits, normalized IOS values were assessed as the signal:noise ratio $(\mathrm{S}: \mathrm{N})$. The intensity of IOS in active cortical columns as a ratio of that in the surrounding inactive neural tissue was calculated as the ratio of the intensities of pixels in the IOS mask to the intensity of luminescence in other recorded parts of the cortex not involved in the cortical response. The spatial $\mathrm{S}: \mathrm{N}$ was $11.4 \pm$ $0.8 \mathrm{~dB}$. However, the spatial $\mathrm{S}: \mathrm{N}$ did not reflect changes in luminescence intensity in response to stimulation of the sensory input; for this purpose we calculated the normalized IOS level using the above formula. Results of analysis of the amplitude characteristics of IOS in the active cortical column showed that IOS amplitude reached $0.13 \pm 0.02 \%$ of baseline amplitude recorded before stimulation (Fig. 2).

These data led to the conclusion that a shift in the illumination spectrum to the near infrared area provided for effective recording of IOS in rat pups in the first three weeks after birth despite the low amplitude of IOS.

Addition of noise to assess the effectiveness of PCA in the detection of IOS. Automatic detection of IOS using PCA in all cases (15 animals) coincided with the mask of the IOS area identified by the operator. Overlap of the manual and automatic detection areas was $88.3 \pm 3.6 \%$. Gradual addition of noise to the video signal containing IOS using additive normal noise showed that PCA effectively detected the position of IOS at $\sigma=4$; subsequent increases led to decreases in the probability of correct detection and, when $\sigma=10$ was exceeded, the probability of detecting IOS was 0 . In parallel with assessment of detection accuracy, the area of coincidence of automatic and manual detection of IOS was analyzed. When there was no added noise, the area of overlap was $60 \%$; addition of noise led to a decrease in 
the area of overlap, due to a large extent to decreases in the sizes of the areas of automatic IOS detection, though complete disappearance of overlap between intrinsic optical signals obtained by the operator and by using PCA occurred at $13 \sigma$ for addition of noise. It should be noted that exceeding the $4 \sigma$ threshold the operator had difficulty identifying the area of the corresponding IOS, while use of PCA provided successful determination of IOS in almost half the cases, its overlap area corresponding to half the area of IOS in controls selected by the operator using the baseline signal.

Thus, these results provide evidence that the PCA method is highly effective in detecting IOS in the immature brain. PCA has high sensitivity, which is apparent in terms of its applicability to very noisy video signals containing IOS.

Discussion. Comparison of red and near infrared light for detection of IOS. Recording of IOS in neurobiology is usually performed using red light $(600-700 \mathrm{~nm})$, because of the absorption spectra of oxy- and deoxyhemoglobin. The finding of differences between the absorption spectra of the two forms of hemoglobin provides for quantitative assessment of the uptake of blood oxygen by neural tissue during activity [11]. Preference in clinical investigations and diagnostic procedures is given to the near infrared spectrum, because of the greater penetrating ability of infrared light. Recording of IOS in humans requires light to penetrate bone and soft tissues on its way to the cerebral cortex and back. As differences in the absorption spectra of oxy- and deoxyhemoglobin are lower in the near infrared spectrum, assessment of oxygen consumption by neurons is more difficult. Preference in our experiments was given to infrared light for the following reasons. The shift to longer wavelengths (700-900 nm) for recording of IOS in neonatal rat pups, as opposed to the widely used red light $(600-700 \mathrm{~nm})$, significantly decreased the number of artifacts, which was associated primarily with the existence of an "optical window" in which the soft tissues and skull bones are almost transparent, which helps decrease the contribution of blood flow to IOS. However, the overall effect occurring as a result of changes to the optical properties of active neural tissue and absorption of light in the infrared range due to changes in the ratio of oxy- and deoxyhemoglobin allowed IOS to be detected - as darkening in the area containing the active cortical column. Thus, use of the near infrared spectrum at a wavelength of $850 \mathrm{~nm}$ gave better detection of IOS than using red light with a wavelength of $610 \mathrm{~nm}$ (data not shown, see [12]).

Principal components analysis for detection of IOS in the immature brains of neonatal rats. PCA is a method widely used to decrease the dimensionality of data with minimal losses of information. These properties are required for extraction of useful signals from noisy data with low S:N. Thus, PCA is a priority choice for detecting IOS in the adult brain, where the amplitude of evoked IOS rarely reaches low percentages $[1,2]$. Low IOS levels led to the development and introduction of other analytical methods, such as independent components analysis [6], indicator functions [4], truncated differences [5], and extended spatial decorrelation $[13,14]$. These methods are very effective for extracting weak signals from noisy data, though they are complex and require massive mathematical processing, limiting their use. Thus, from the point of view of the simplicity of use, the choice of method allowing IOS to be detected and extracted from noisy data falls on PCA. However, most studies using PCA to detect IOS have been performed in adult animals, while the immature brain, which is of particular interest for developmental neurobiology, remains poorly studied. In the present study, we obtained the first demonstration that the use of PCA allows the locations of active neural tissue in the somatosensory cortex of neonatal rats to be identified.

The results obtained here lead to the following conclusions:

1. As compared with red light $(610 \mathrm{~nm})$, use of near-infrared light $(850 \mathrm{~nm})$ gave better detection of IOS in the immature brains of rat pups. The increase in contrast between the active cortical column with IOS in the surrounding inactive neural tissue came from both an increase in the depth of penetration of near-infrared light and a decrease in the contribution of artifacts due to the hemovascular component, which has maximal absorption in the green part of the visible spectrum but also adds noise in the red range.

2. The principal components analysis method can be used with success to detect IOS in the immature brain. In contrast to other methods used for detecting IOS in adult tissue, the PCA method does not require large computation resources and is optimal for the immature brain.

3. Controlled addition of noise to the signal demonstrated the high effectiveness of the PCA method for detecting IOS in neonatal rat pups, pointing to the potential of applying IOS-based functional mapping of the developing brain using the PCA method.

This study was supported financially by the Russian Scientific Foundation in the framework of Program No. 1615-10174, "Mapping of the developing brain by recording intrinsic optical signals."

\section{REFERENCES}

1. A. Grinvald, R. D. Frostig, E. Lieke, and R. Hildesheim, "Optical imaging of neuronal activity," Physiol. Rev., 4, No. 4, 1285-1366 (1988).

2. R. D. Frostig, E. E. Lieke, D. Y. Ts'o, and A. Grinvald, "Cortical functional architecture and local coupling between neuronal activity and the microcirculation revealed by in vivo high-resolution optical imaging of intrinsic signals," Proc. Natl.Acad. Sci. USA, 87, No. 16, 6082-6086 (1990).

3. M. Sintsov, D. Suchkov, R. Khazipov, and M. Minlebaev, "Improved recordings of the optical intrinsic signals in the neonatal rat barrel cortex," BioNanoScience (2016), doi: 10.1007/S12668.016.0359.

4. R. M. Everson, A. K. Prashanth, M. Gabbay, et al., "Representation of spatial frequency and orientation in the visual cortex," Proc. Natl. Acad. Sci. USA, 95, No. 14, 8334-8338 (1998).

5. M. Gabbay, C. Brennan, E. Kaplan, and L. Sirovich, "A principal components-based method for the detection of neuronal activity 
maps: application to optical imaging," Neuroimage, 11, No. 4, 313 325 (2000)

6. A. Hyvarinen and E. Oja, "Simple neuron models for independent components analysis," Int. J. Neural Syst., 7, No. 6, 671-687 (1996).

7. R. Khazipov, D. Zaynutdinova, E. Ogievetsky, et al., "Atlas of the Postnatal Rat Brain in Stereotaxic Coordinates," Front. Neuroanat., dx.doi.org./103389/fnana.2015.00161.2015.

8. O. Mitrukhina, D. Suchkov, R. Khazipov, and M. Minlebaev, "Imprecise whisker map in the neonatal rat barrel cortex," Cereb. Cortex, doi: 10.1093/cercor/bhu169.2014.

9. C. H. Chen-Bee, D. B. Polley, B. Brett-Green, et al., "Visualizing and quantifying evoked cortical activity assessed with intrinsic signal imaging," J. Neurosci. Meth., 97, No. 2, 157-173 (2000).
10. A. M. O'Farrell, D. E. Rex, A. Muthialu, et al., "Characterization of optical intrinsic signals and blood volume during cortical spreading depression," Neuroreport, 11, No. 10, 2121-2125 (2000).

11. S. Nelson, L. Toth, B. Sheth, and M. Sur, "Orientation selectivity of cortical neurons during intracellular blockade in inhibition," Science, 265, 774-777 (1994).

12. M. Minlebaev, M. Colonnese, T. Tsintsadze, et al., "Early gamma oscillations synchronize developing thalamus and cortex," Science, 334, No. 6053, 226-229 (2011).

13. M. Stetter, I. Schiessl, T. Otto, et al., "Principal component analysis and blind separation of sources for optical imaging of intrinsic signals," Neuroimage, 11, 482-490 (2000).

14. R. Everson, B. W. Knight, and L. Sirovich, "Separating spatially distributed response to stimulation from background. I. Optical imaging," Biol. Cybern., 77, No. 6, 407-417 (1997). 